\title{
Application of Beta-lactam Therapeutic Drug Monitoring in Clinical Practice Using HPLC
}

\author{
Toshiharu Urakami ${ }^{1,2}$, Yosuke Aoki ${ }^{3 *}$, Mami Fukuoka ${ }^{3,4}$, Hiroki Magarifuchi ${ }^{3}$, Masaki Nagata ${ }^{3}$, Zenzo Nagasawa ${ }^{5}$, Yukitaka Nakano $^{2}$ and
} Hiroshi Fujito ${ }^{2}$

${ }^{1}$ Department of Pharmacy, Saga Prefectural Hospital KOSEIKAN, Saga, Japan

${ }^{2}$ Department of Clinical Pharmaceutics, Saga University Graduate School of Medical Science, Saga, Japan

${ }^{3}$ Division of Infection Control and Prevention, Saga University Hospital, Saga, Japan

${ }^{4}$ Division of Infection Control and Prevention, Saga Prefectural Hospital KOSEIKAN, Saga, Japan

${ }^{5}$ Department of Laboratory Medicine, Saga University Hospital, Saga, Japan

\begin{abstract}
In beta-lactam therapy, investigation of the pharmacokinetic-pharmacodynamic (PK-PD) relationship has provided surrogate makers to predict clinical outcome. This study was designed to verify the therapeutic efficacy and clinical utility of beta-lactam therapeutic drug monitoring (TDM) in critically ill patients using high-performance liquid chromatography (HPLC). This cohort study included 13 patients who were intravenously administered ceftazidime ( $\mathrm{n}$ $=6)$, cefepime $(n=1)$, imipenem $(n=1)$, meropenem $(n=1)$, or piperacillin $(n=4)$. Blood samples were collected at 3 time points fitted to a 1-compartment model, and concentrations were determined using HPLC. The PK-PD target was the percentage of the dosing interval during which the antibiotic concentration exceeded the minimum inhibitory concentration for the pathogens (\%T > MIC), which is $50 \%$ for penicillins (piperacillin), $60 \%$ for cephalosporins (ceftazidime and cefepime), and $40 \%$ for carbapenems (imipenem and meropenem). Our process using HPLC enables the analytical results of TDM to be available within half a day. The results revealed significant inter-patient pharmacokinetic variability. During the initial regimen, beta-lactam concentrations reached the target \% $>$ MIC in all patients, and dosage reduction was required for 5 patients (38\%). Of the 13 evaluable patients, clinical improvement was observed in $11(85 \%)$, and microbiological success was observed in $10(77 \%)$. In summary, beta-lactam TDM achieved the treatment goals and might also allow for the personalization to prevent overdosing for variable pharmacokinetic changes in critically ill patients. These findings indicate that beta-lactam TDM using HPLC can be used in the standard pharmacy clinical practice for critically ill patients.
\end{abstract}

Keywords: High-performance liquid chromatography (HPLC); Beta-lactam; Pharmacokinetics- pharmacodynamics (PK-PD); Therapeutic drug monitoring (TDM); Standard pharmacy clinical practice

\section{Introduction}

Beta-lactams are administered as first-line therapy for the majority of infections. The pharmacokinetic-pharmacodynamic (PK-PD) theory indicates that the antibacterial activity of these antibiotics is timedependent. Dr. Craig and colleagues demonstrated that the percentage of time of the dosing interval during which the antibiotic concentration exceeds the minimum inhibitory concentration for pathogens $(\% \mathrm{~T}>$ MIC) was linked to the antibiotics' microbiological and clinical effects in vivo in animals and humans and in vitro [1]. Thus, to achieve a bactericidal effect, laboratory and clinical evidence indicates that the $\% \mathrm{~T}>$ MIC should be $50 \%$ for penicillins, $60 \%$ for cephalosporins, and $40 \%$ for carbapenems; these appear to be the PK-PD target values for individualized dosage regimens $[1,2]$.

Optimizing the antibiotic regimen should be considered as a critical strategy to maximize therapeutic success while minimizing toxicity and the development of resistance for infectious disease patients [3,4]. Traditionally, although glycopeptides and aminoglycosides are applied to therapeutic drug monitoring (TDM) to prevent toxicity, this approach has not been widely applied to beta-lactams because of their safety. In beta-lactam therapy, an understanding of the PKPD relationship has provided surrogate makers to predict the clinical outcome and indicated that it is increasingly necessary to individualize antimicrobial regimen to achieve the optimal antimicrobial treatment rather than to avoid toxicity.

For the clinical application of the PK-PD theory for beta-lactams, a continuous or long-term infusion of beta-lactams has been used because the time-dependent antibiotic activity associated with this approach was superior to a short-term infusion over the last decade $[5,6]$. However, these studies did not use individually targeted approaches based on the actual serum concentration of beta-lactams and their pharmacokinetics. Although the best clinical practice is to estimate the pharmacokinetic parameters for each patient, the implementation of beta-lactam TDM based on Craig's indices has yet to be investigated in the clinical practice [7]. The aim of this study was to validate the clinical utility of patient-specific beta-lactam TDM in pharmacy clinical practice.

\section{Methods}

\section{Patients}

Patients were included in the study if (1) they had an indication to receive beta-lactams intravenously as a definite therapy, and (2) beta-lactam TDM was encouraged by infectious disease physicians for critical infections. The exclusion criteria included patients aged

*Corresponding author: Yosuke Aoki, Division of Infection Control and Prevention, Saga University Hospital, 5-1-1, Nabeshima, Saga 849-8501, Japan, Tel: +81-95234-3242; Fax: +81-952-32-3294; E-mail: aokiy3@cc.saga-u.ac.jp

Received June 21, 2011; Accepted July 22, 2011; Published July 28, 2011

Citation: Urakami T, Aoki Y, Fukuoka M, Magarifuchi H, Nagata M, et al. (2011) Application of Beta-lactam Therapeutic Drug Monitoring in Clinical Practice Using HPLC. J Bioanal Biomed S3: 001. doi:10.4172/1948-593X.S3-001

Copyright: $\odot 2011$ Urakami T, et al. This is an open-access article distributed under the terms of the Creative Commons Attribution License, which permits unrestricted use, distribution, and reproduction in any medium, provided the original author and source are credited. 
$<20$ years, a history of an allergy to any beta-lactam, pregnancy and lactation. As a result, 13 patients ( 9 males and 4 females; median age, 62 years; interquartile age range, 58 - 80 years; mean \pm standard deviation (SD) actual body weight, $61.2 \pm 13.5 \mathrm{~kg}$ ) were enrolled in this study. This study was a prospective cohort clinical trial carried out from February 2009 to December 2010. The study protocol was approved by the Scientific and Ethics Committees of Saga University Hospital and submitted for review. Written informed consent was obtained from each patient or first-degree relative if the patient was unable to give informed consent owing to his or her condition. We collected demographic and laboratory data (age, gender, weight and serum creatinine). Creatinine clearance was estimated using the commonly used Cockcroft-Gault equation (Cockcroft CLcr).

\section{Determination of beta-lactam concentrations}

The following beta-lactams were subjected to TDM: ceftazidime (CAZ), cefepime (CFPM), imipenem (IPM) in combination with cilastatin, meropenem (MEPM) and piperacillin (PIPC; either alone or in combination with tazobactam). The initial dosage was chosen empirically. Standard infusion times for beta-lactams at Saga University Hospital are $0.5-1.0$ h. Samples $(5 \mathrm{~mL})$ of venous blood for all beta-lactams were collected to determine the antibiotic concentration at the 3 following time points: prior to administration (trough level, within 10 min before drug administration) and at 2 other time points at least $30 \mathrm{~min}$ apart after the end of drug administration (during the elimination phase). The exact sampling time was recorded by medical staff. Blood samples were centrifuged at $5000 \times \mathrm{g}$ for 10 min. The obtained plasma was centrifuged in tubes containing filters with a cut-off of approx. $10 \mathrm{kDa}$ (Microcon Ultracel YM-10; Millipore, Bedford, USA) in a fixed-angle rotor at $14000 \times \mathrm{g}$ at $37^{\circ} \mathrm{C}$ for $10 \mathrm{~min}$. The ultrafiltrate concentrations of free beta-lactam in the serum were determined by high-performance liquid chromatography (HPLC), as previously described with a slight modification [8-10]. As the protein binding rate is low for these beta-lactams, the free concentration is not significantly different from the total concentration [11].

\section{Pharmacokinetic-pharmacodynamic analyses}

Pharmacokinetic parameters were individually derived for each antibiotic and each subject. The serum concentration data (3 points) were fitted to a 1-compartment model. Using the Sawchuk-Zaske method, the pharmacokinetic parameters were determined using systemic clearance $(\mathrm{CL})$ in $\mathrm{mL} \cdot \mathrm{min}^{-1}$, volume of distribution $(\mathrm{Vd})$ in $\mathrm{L}$, and elimination half-life $\left(\mathrm{t}_{1 / 2}\right)$ in $\mathrm{h}$. Descriptive statistics (mean $\pm \mathrm{SD}$ ) were used to summarize the pharmacokinetic parameters of CAZ and PIPC. In each patient, the profile of the concentration-time curve was simulated using patient-specific pharmacokinetic values, and the $\% \mathrm{~T}>$ MIC from $0 \mathrm{~h}$ (time of administration) to $24 \mathrm{~h}$ was calculated by using a single-dose infusion model with the following equation [12]:

$\% \mathrm{~T}>\mathrm{MIC}=\left\{\mathrm{T}_{\text {inf }}\left[\ln \left(\frac{\operatorname{Rinf} / \mathrm{CL}}{\operatorname{Rinf} / \mathrm{CL}-\mathrm{MIC}}\right)+\ln \left(\frac{\operatorname{Rinf}}{\mathrm{CL}}\right)-\ln (\mathrm{MIC})\right] \times \frac{1}{\mathrm{kel}}\right\} \times \frac{100}{\tau}$

This equation assumes that the peak concentration at the end of the infusion is calculated as $\mathrm{R}_{\text {inf }} / \mathrm{CL}$. $\mathrm{T}_{\text {inf }}$ is the infusion time in $\mathrm{h}, \mathrm{R}_{\mathrm{inf}}$ is the infusion rate in $\mathrm{mg} \cdot \mathrm{h}^{-1}, \mathrm{CL}$ is the clearance rate in $\mathrm{L} \cdot \mathrm{h}^{-1}, \mathrm{k}_{\mathrm{e}}$ is the rate constant, and $\tau$ is the dosing interval in $\mathrm{h}$. MICs for the pathogens were determined using the microdilution method. Dosage adjustment or frequency increase was performed when the $\% \mathrm{~T}>$ MIC was below $50 \%$ for PIPC, $60 \%$ for CAZ and CFPM and $40 \%$ for IPM and MEPM against each pathogen (bactericidal exposures). Because the serum concentration of beta-lactams does not have to remain above the MIC for the entire dosing interval in order to achieve the maximal effect, a lower dose can be used to produce an equivalent effect with lower adverse events. So, according to the pharmacokinetic model, if serum concentrations were enough too high, initial dosage was reduced to maintain bactericidal exposures. TDM was performed only once per patient.

Descriptive statistics were calculated using the SYSTAT version 10.2 software (Systat Software, Inc., Chicago, IL, USA).

\section{Response to therapy}

The efficacy variables were clinical and bacteriological responses. The clinical response was categorized as cure, failure or undetermined by infectious disease physicians. A cure was comprehensively defined as diminishing signs and symptoms. The bacteriological response was categorized as eradication, persistence or undetermined by bacterial cultures. An eradication was defined as over 1 set of negative follow-up screenings.

\section{Results and Discussion}

\section{The use of beta-lactam TDM by HPLC in the clinical setting}

To our knowledge, this is the study to describe the use of betalactam TDM in the daily clinical practice for critically ill patients using HPLC. Generally, there are some significant hurdles with beta-lactam TDM, for example the fact that HPLC has a slow turnaround time. Using ultrafiltration, we can simply measure free (pharmacologically active) beta-lactam concentrations for less than several hours. Another hurdle is the complexity of pharmacokinetic analysis. We calculated the individual pharmacokinetic profile of beta-lactam using a 1-compartment model, which is the most acceptable pharmacokinetic model for use in the clinical setting. Some antibiotic PK-PD trials employed multi-compartment models, but any model was employed the same as a non-compartmental model $[13,14]$. Our process enables the analytical results of TDM to be available within half a day.

\section{Patients}

Total characteristics of the patients are shown in Table 1. Six patients were treated with CAZ, 1 with CFPM, 1 with IPM, 1 with MEPM, and 4 with PIPC. Four patients (Patient 2, 3, 5 and 13; Table 1) had renal dysfunction (Cockcroft CLcr $<50 \mathrm{~mL} \cdot \mathrm{min}^{-1}$ ) and 5 patients were receiving renal replacement therapy (continuous venovenous hemodiafiltration, Patient 4, 9, 10 and 11; hemodialysis, Patient 12; Table 1). The infecting pathogens were 14 gram-negative bacilli and 1 gram-positive coccus. In these strains, the MIC of the beta-lactams ranged from 0.5 to $8 \mathrm{mcg} \cdot \mathrm{mL}^{-1}$. The infectious diseases requiring betalactam therapy were as follows: bacteremia $(n=6)$, pneumonia $(n=3)$, pyelonephritis $(n=2)$, peritonitis $(n=1)$, and prosthetic joint infection $(\mathrm{n}=1)$.

\section{Pharmacokinetics and pharmacodynamics}

All blood samples were collected in non-anticoagulant (red top) tubes and immediately analyzed. For PIPC, the following pharmacokinetic parameters were noted: $\mathrm{t}_{1 / 2}=3.1 \pm 1.3 \mathrm{~h}, \mathrm{Vd}=22.2$ $\pm 11.2 \mathrm{~L}$, and $\mathrm{CL}=95.2 \pm 15.1 \mathrm{~mL} \cdot \mathrm{min}^{-1}$. For CAZ, the following pharmacokinetic parameters were noted: $\mathrm{t}_{1 / 2}=17.6 \pm 16.6 \mathrm{~h}, \mathrm{Vd}=13.5$ $\pm 5.6 \mathrm{~L}$, and $\mathrm{CL}=24.7 \pm 34.5 \mathrm{~mL} \cdot \mathrm{min}^{-1}$, respectively. A comparison of the PIPC and CAZ data obtained in this study versus the published data $[15,16]$ is given in Table 2. In our patients, the PIPC mean $t_{1 / 2}$, $\mathrm{k}_{\mathrm{el}}$, Vd and CL were 3.9-fold greater, 3-fold smaller, 2.1-fold greater 
Citation: Urakami T, Aoki Y, Fukuoka M, Magarifuchi H, Nagata M, et al. (2011) Application of Beta-lactam Therapeutic Drug Monitoring in Clinical Practice Using HPLC. J Bioanal Biomed S3: 001. doi:10.4172/1948-593X.S3-001

Page 3 of 5

\begin{tabular}{|c|c|c|c|c|c|c|c|c|c|c|c|}
\hline Patient & Age (yr)/ & Weight & Cockcroft & \multirow{2}{*}{ Diagnosis } & Pathogen / & \multirow{2}{*}{ Beta-lactam } & \multirow{2}{*}{$\begin{array}{l}\text { Initial } \\
\text { dosage }\end{array}$} & \multirow{2}{*}{$\begin{array}{l}\text { Dosage } \\
\text { change }\end{array}$} & Initial & Clinical & Microbiological \\
\hline No & Gender & $(\mathrm{kg})$ & $\mathrm{CL}_{\mathrm{cr}}\left(\mathrm{mL} \cdot \mathrm{min}^{-1}\right)$ & & $\mathrm{MIC}\left(\mathrm{mg} \cdot \mathrm{L}^{-1}\right)$ & & & & $\% \mathrm{~T}>\mathrm{MIC}$ & efficacy & efficacy \\
\hline 1 & $59 / \mathrm{M}$ & 62.2 & 76.1 & Pneumonia & P.a / 8 & $\mathrm{CAZ}$ & $2 \mathrm{~g}, \mathrm{q} 8 \mathrm{~h}$ & $1 \mathrm{~g}, \mathrm{q} 3 \mathrm{~h}$ & 100 & Cure & Eradication \\
\hline 2 & $81 / M$ & 64.7 & 33.6 & Bacteremia & E.cl. / 0.5 & MEPM & $0.5 \mathrm{~g}, \mathrm{q} 6 \mathrm{~h}$ & $0.5 \mathrm{~g}, \mathrm{q} 12 \mathrm{~h}$ & 100 & Failure & Undetermined \\
\hline 3 & $83 / \mathrm{M}$ & 43.6 & 20 & Pyelonephritis & P.a. / 2 & CAZ & $2 g, q 24 h$ & $1 \mathrm{~g}, \mathrm{q} 12 \mathrm{~h}$ & 100 & Cure & Eradication \\
\hline 4 & $58 / \mathrm{F}$ & 60 & CVYHDF & Peritonitis & $F$ & IPM & $05 a-12 b$ & $05 a \Omega 2 \Delta h$ & 100 & Cure & Frodingtion \\
\hline 4 & $50 / 5$ & 00 & CVVHDF & Peritonitis & E.co. 10.5 & with cirastatin & $0.5 \mathrm{~g}, \mathrm{q} T 2 \mathrm{n}$ & $0.5 \mathrm{~g}, \mathrm{q} 24 \mathrm{n}$ & 100 & Cure & Eradication \\
\hline 5 & $80 / F$ & 41.8 & 6.85 & Bacteremia & E.co. / 0.5 & CAZ & $2 g, q 24 h$ & $1 \mathrm{~g}, \mathrm{q} 24 \mathrm{~h}$ & 100 & Undetermined & Eradication \\
\hline 6 & $54 / \mathrm{M}$ & 67.2 & 143 & Bacteremia & P.a. / 8 & CFPM & $1 \mathrm{~g}, \mathrm{q} 8 \mathrm{~h}$ & No & 58 & Cure & Eradication \\
\hline 7 & $21 / M$ & 042 & 195 & Recteromia & S.d. $/ 2$ & PIPC & $4 a \mathrm{afh}$ & No & 100 & Cure & Fradication \\
\hline$I$ & $217 \mathrm{IV}$ & 94.2 & 195 & Dacteremia & E. co / 2 & FirC & $4 \mathrm{~g}, 4011$ & INO & 100 & cure & ETauication \\
\hline 8 & $84 / F$ & 526 & 669 & Renal abscess & $F \operatorname{col} 12$ & PIPC & $45 a g 8 h$ & $\mathrm{No}$ & 100 & Failure & Persistence \\
\hline 0 & 0411 & $0<.0$ & 00.0 & Bacteremia & L.60.1 2 & with tazobactam & $4.09,4011$ & INo & 100 & ramure & 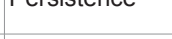 \\
\hline 9 & $58 / \mathrm{M}$ & 62 & CVVHDF & Pvelonephritis & $E \mathrm{co} / 2$ & PIPC & $-4.5 \mathrm{~g} g 8 \mathrm{~h}$ & $\mathrm{No}$ & 100 & Cure & Hndetermined \\
\hline & & & & & & with tazobactam & $4.5 \mathrm{~g}, \mathrm{yon}$ & NO & 100 & cure & Undetermined \\
\hline 10 & $82 / \mathrm{M}$ & 74.3 & CVVHDF & Pneumonia & $\mathrm{E} \mathrm{cl} / 2$ & PIPC & $45 a-a 12 h$ & No & 100 & Cure & Undetermined \\
\hline & & & & & & with tazobactam & & & & Cure & Undetermined \\
\hline 11 & $62 / M$ & 61.2 & CVVHDF & Bacteremia & C.k. / 1 & $\mathrm{CAZ}$ & $2 \mathrm{~g}, \mathrm{q} 12 \mathrm{~h}$ & $1 \mathrm{~g}, \mathrm{q} 12 \mathrm{~h}$ & 100 & Cure & Eradication \\
\hline 12 & $58 / \mathrm{M}$ & 51.1 & IHD & Prosthetic joint & P.a. $/ 4$ & CAZ & 19 after IHD & No & 100 & Undetermined & Eradication \\
\hline & & & & Infection & & CAL & 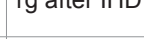 & INO & 100 & Ondieterminted & Lianitumiti \\
\hline 13 & $78 / F$ & 60.7 & 43.1 & Pneumonia & P.a. / 1, A.b. / 4 & CAZ & $2 g, q 12 h$ & $1 \mathrm{~g}, \mathrm{q} 12 \mathrm{~h}$ & 100 & Cure & Eradication \\
\hline
\end{tabular}

Cockcroft CLcr, Creatinine clearance caluculated according to the Cockcroft-Gault formula; MIC, minimum inhibitory concentration; \%T>MIC, the percentage of time of the dosing interval during which the antibiotic concentration exceeds the MIC for the pathogens; CVVHDF, continuous venovenous hemodiafiltration; IHD, intermittent hemodialysis; CAZ, ceftazidime; MEPM, meropenem; IPM, imipenem; CFPM, cefepime; PIPC, piperacillin;

P.a., Pseudomonas aeruginosa; E.cl., Enterobacter cloacae; E.co., Escherichia coli; S.d., Streptococcus dysgalactiae; C.k., Citrobactor koseri; A.b., Acinetobacter baumannii

Table 1: Patient characteristics of 13 patients.

\begin{tabular}{|l|l|l|l|l|}
\hline \multirow{2}{*}{ PK Parameter } & \multicolumn{2}{|l|}{ Piperacillin (Mean $\pm \mathrm{SD})$} & \multicolumn{2}{l|}{ Ceftazidime (Mean \pm SD ) } \\
\cline { 2 - 4 } & This study & Occhipinti et al. [15] & \multicolumn{2}{|l}{ This study } \\
\hline $\mathrm{t}_{1 / 2}(\mathrm{~h})$ & $3.1 \pm 1.3$ & $0.8 \pm 0.1$ & $17.6 \pm 16.6$ \\
\hline Vd $(\mathrm{L})$ & $22.2 \pm 11.2$ & $10.5 \pm 1.4$ & $1.9 \pm 0.2$ & $13.5 \pm 5.6$ \\
\hline $\mathrm{CL}\left(\mathrm{mL} \cdot \mathrm{min}^{-1}\right)$ & $95.2 \pm 15.1$ & $182.0 \pm 20.0$ & $24.7 \pm 34.5$ \\
\hline
\end{tabular}

$\mathrm{SD}$, standard deviation; $\mathrm{t} 1 / 2$, elimination half-time;

$\mathrm{Vd}$, volume of distribution; $\mathrm{CL}$, total drug clearance

Table 2: Comparison of piperacillin and ceftazidime pharmacokinetic parameters in this study versus published data for healthy volunteers.

\begin{tabular}{|l|l|l|l|l|l|}
\hline Beta-lactam & Patients & No revision & Increased dosing frequency & Dose increased \\
\hline Ceftazidime & $6(46 \%)$ & $1(17 \%)$ & $1(17 \%)$ & $0(0 \%)$ \\
\hline Cefepime & $1(8 \%)$ & $1(100 \%)$ & $0(0 \%)$ & $0(0 \%)$ & 0 \\
\hline Imipenem & $1(8 \%)$ & $1(100 \%)$ & $0(0 \%)$ & $0(0 \%)$ & $0(0 \%)$ \\
\hline Meropenem & $1(8 \%)$ & $0(0 \%)$ & $0(0 \%)$ & $0(0 \%)$ & 0 \\
\hline Piperacillin & $4(30.8 \%)$ & $4{ }^{b}(100 \%)$ & $0(0 \%)$ & $1(100 \%)$ & $0(0 \%)$ \\
\hline Total & $13(100 \%)$ & $7(54 \%)$ & $1(8 \%)$ & $0(0 \%)$ & \\
\hline
\end{tabular}

aDosing frequency increase include dose not change

bIncludes Patient 8

Table 3: Result of dose adjustment following beta-lactam therapeutic drug monitoring

and 1.9-fold smaller, respectively, and the CAZ mean $\mathrm{t}_{1 / 2}, \mathrm{Vd}$ and CL were 9.3-fold greater, equal and 4.7-fold smaller, respectively, than the values for healthy volunteers. Today, only a small number of patients are assessed in pharmacokinetic studies. Monte Carlo simulation has replaced these approaches to attain PK-PD targets against specific pathogens to predict the clinical outcome $[17,18]$. Therefore population pharmacokinetic parameters are essential for Monte Carlo simulation. Population pharmacokinetic analyses have been generally determined in healthy volunteers, since several studies have indicated that there is no difference between healthy volunteers and patients in terms of pharmacokinetic profiles [17,19]. Nevertheless, true between-patient variability was observed in our patients. In addition, the pharmacokinetic profiles of PIPC and CAZ in this study were significantly different from those in the healthy volunteers. This significant difference demonstrates the benefit of the monitoring on actual serum concentrations and pharmacokinetics for each patient. Furthermore, for renal elimination of antibiotics, it is difficult to precisely estimate the pharmacokinetic parameters for critically ill patients by using population pharmacokinetic analysis (e.g., Bayesian estimation), because correct Cockcroft CLcr values cannot be obtained in the case of critical illness [20,21]. So given the beta-lactam pharmacokinetics without Cockcroft CLcr, TDM appears to be a valuable method [22].

The $\% \mathrm{~T}>\mathrm{MIC}$ for each individual patient are shown in Table 1. Certainly, the beta-lactam serum concentrations in many patients were high enough because of renal function impairments as well as relating low MICs for the pathogens encountered in this study. Consequently, all of the patients in this study achieved the target value with an 
empirical dosage. The $\% \mathrm{~T}>\mathrm{MIC}$ for Patient 6 was considered to be approximately $60 \%$. Clinical situations are particularly heterogeneous; therefore, treatment individualization must ensure the best match between the pharmacokinetic and pharmacodynamic properties. Thus, TDM appears to be a valuable method for beta-lactam therapy.

\section{Dose individualization}

Initial dosage and dosage change for each individual patient are shown in Table 1, and the effects of beta-lactam prescribed for dose adjustment during TDM are described in Table 3. In 1 patient (Patient 3; $8 \%$ of patients), the dosage per day was maintained, but the dosing frequency was increased. In 5 patients (Patients 1, 2, 5, 11, and 13;38\% of patients), antibiotic levels were considered to be too high, and the beta-lactam dosages were therefore reduced. No patients received an increased dosage. Dr. Blondiaux and colleagues reported that TDMguided dose adjustment was required in $50 \%$ of patients for the continuous infusion of PIPC in combination with tazobactam [23]. Similarly, in this study, the patients required a correction of their initial regimens following TDM and the most of them were successful in decrease beta-lactam dosages in the present study. These results suggest that TDM-driven dose personalization is required for beta-lactams in clinical practice.

Eleven of the 13 patients (85\%) were clinically evaluable and $10 / 13$ patients $(77 \%)$ were also microbiologically evaluable. Success was achieved in 9/11 (82\%) clinically evaluable patients and in 9/10 (90\%) microbiologically evaluable patients. A few clinical studies have attempted to evaluate the relationship between PK-PD targets and clinical success. Dr. Scaglione reported that the treatment failure rate was $17.5 \%$ during TDM approaches for antibiotics of various categories [24]. Dr. Roberts and colleagues suggested the use of trough concentrations as another PK-PD target at $4-5 \times$ the concentration of the MIC for the pathogen and reported that the clinical success rate was $87.3 \%$ during beta-lactam TDM [25]. As observed by Dr. Roberts and colleagues, contrasting target values of $\% \mathrm{~T}>\mathrm{MIC}$ were recently reported by some authors $[26,27]$. The very limited pertinent literature for beta-lactam TDM does not allow a direct comparison between our evaluation and those of previous trials; however, the results from the available reports and the present study are equivalent and suggest that beta-lactam TDM is useful to successfully personalize treatment. For a more precise success rate, further investigations are necessary to determine the optimal targets for $\% \mathrm{~T}>\mathrm{MIC}$.

\section{Conclusion}

In daily clinical practice, beta-lactam TDM using HPLC achieved the treatment goals and might also allow for the personalization to prevent overdosing for variable pharmacokinetic changes of critically ill patients. This study had a small sample size; however, these findings indicate that beta-lactam TDM using HPLC can be used in the standard pharmacy care.

\section{References}

1. Craig WA (1998) Pharmacokinetic/pharmacodynamic parameters: rationale for antibacterial dosing of mice and men. Clin Infect Dis 26: 1-10.

2. Drusano GL (2003) Prevention of resistance: a goal for dose selection for antimicrobial agents. Clin Infect Dis 36 (Suppl 1): S42-50.

3. Kollef MH, Sherman G, Ward S, Fraser VJ (1999) Inadequate antimicrobia treatment of infections: a risk factor for hospital mortality among critically ill patients. Chest 115: 462-474.

4. Garnacho-Montero J, Garcia-Garmendia JL, Barrero-Almodovar A, JimenezJimenez FJ, Perez-Paredes C, et al. (2003) Impact of adequate empirical antibiotic therapy on the outcome of patients admitted to the intensive care unit with sepsis. Crit Care Med 31: 2742-2751.

5. Roberts JA, Paratz J, Paratz E, Krueger WA, Lipman J (2007) Continuous infusion of beta-lactam antibiotics in severe infections: a review of its role. Int $J$ Antimicrob Agents 30: 11-18.

6. Kasiakou SK, Lawrence KR, Choulis N, Falagas ME (2005) Continuous versus intermittent intravenous administration of antibacterials with time-dependen action: a systematic review of pharmacokinetic and pharmacodynamic parameters. Drugs 65: 2499-2511.

7. Scaglione F, Paraboni L (2008) Pharmacokinetics/pharmacodynamics of antibacterials in the Intensive Care Unit: setting appropriate dosing regimens. Int J Antimicrob Agents 32: 294-301.

8. Isla A, Arzuaga A, Maynar J, Gascón AR, Solinís MA, et al. (2005) Determination of ceftazidime and cefepime in plasma and dialysate-ultrafiltrate from patients undergoing continuous veno-venous hemodiafiltration by HPLC. J Pharm Biomed Anal 39: 996-1005

9. Kurihara Y, Kizu J, Hori S (2008) Simple and rapid determination of serum carbapenem concentrations by high-performance liquid chromatography. J Infect Chemother 14: 30-34.

10. Arzuaga A, Isla A, Gascón AR, Maynar J, Martín A, et al. (2005) Quantitation and stability of piperacillin and tazobactam in plasma and ultrafiltrate from patients undergoing continuous venovenous hemofiltration by HPLC. Biomed Chromatogr 19: 570-578

11. MacGowan AP, Wise $R$ (2001) Establishing MIC breakpoints and the interpretation of in vitro susceptibility tests. J Antimicrob Chemother 48 (Supp 1): $17-28$.

12. Shea KM, Cheatham SC, Wack MF, Smith DW, Sowinski KM, et al. (2009) Steady-state pharmacokinetics and pharmacodynamics of piperacillin tazobactam administered by prolonged infusion in hospitalised patients. Int $\mathrm{J}$ Antimicrob Agents 34: 429-433

13. Wang H, Zhang B, Ni Y, Kuti JL, Chen B, et al. (2007) Pharmacodynamic targe attainment of seven antimicrobials against Gram-negative bacteria collected from China in 2003 and 2004. Int J Antimicrob Agents 30: 452-457.

14. Kuti JL, Florea NR, Nightingale CH, Nicolau DP (2004) Pharmacodynamics of meropenem and imipenem against Enterobacteriaceae, Acinetobacter baumannii, and Pseudomonas aeruginosa. Pharmacotherapy 24: 8-15.

15. Occhipinti DJ, Pendland SL, Schoonover LL, Rypins EB, Danziger LH, et al. (1997) Pharmacokinetics and pharmacodynamics of two multiple-dose piperacillin-tazobactam regimens. Antimicrob Agents Chemother 41: 2511 2517.

16. Lüthy R, Blaser J, Bonetti A, Simmen H, Wise R, et al. (1982) Comparative multiple-dose pharmacokinetics of cefotaxime, moxalactam, and ceftazidime. Rev Infect Dis 4 (Suppl): S581-S584.

17. Kuti JL, Horowitz S, Nightingale CH, Nicolau DP (2005) Comparison of pharmacodynamic target attainment between healthy subjects and patients for ceftazidime and meropenem. Pharmacotherapy 25: 935-941.

18. Kuti JL, Dandekar PK, Nightingale CH, Nicolau DP (2003) Use of Monte Carlo simulation to design an optimized pharmacodynamic dosing strategy for meropenem. J Clin Pharmacol 43: 1116-1123.

19. Ariano RE, Nyhlén A, Donnelly JP, Sitar DS, Harding GK, et al. (2005) Pharmacokinetics and pharmacodynamics of meropenem in febrile neutropenic patients with bacteremia. Ann Pharmacother 39: 32-38.

20. Martin JH, Fay MF, Udy A, Roberts J, Kirkpatrick C, et al. (2010) Pitfalls of using estimations of glomerular filtration rate in an intensive care population. Intern Med J Epub.

21. Hoste EA, Damen J, Vanholder RC, Lameire NH, Delanghe JR, et al. (2005) Assessment of renal function in recently admitted critically ill patients with normal serum creatinine. Nephrol Dial Transplant 20: 747-753.

22. Aoki Y, Urakami T, Magarifuchi H, Nagasawa Z, Nagata M, et al. (2010) The importance of pharmacokinetic consultation of cefepime treatment fo Pseudomonas aeruginosa bacteremia: a case report of severe thermal burn injury. J Infect Chemother 17: 407-411.

23. Blondiaux N, Wallet F, Favory R, Onimus T, Nseir S, et al. (2010) Daily serum piperacillin monitoring is advisable in critically ill patients. Int $\mathrm{J}$ Antimicrob Agents 35: 500-503. 
Citation: Urakami T, Aoki Y, Fukuoka M, Magarifuchi H, Nagata M, et al. (2011) Application of Beta-lactam Therapeutic Drug Monitoring in Clinical Practice Using HPLC. J Bioanal Biomed S3: 001. doi:10.4172/1948-593X.S3-001

Page 5 of 5

24. Scaglione $F(2002)$ Can PK/PD be used in everyday clinical practice. Int $J$ Antimicrob Agents 19: 349-353.

25. Roberts JA, Ulldemolins M, Roberts MS, McWhinney B, Ungerer J, et al. (2010) Therapeutic drug monitoring of beta-lactams in critically ill patients: proof of concept. Int J Antimicrob Agents 36: 332-339.

26. McKinnon PS, Paladino JA, Schentag JJ (2008) Evaluation of area under the inhibitory curve (AUIC) and time above the minimum inhibitory concentration ( $\mathrm{T}>\mathrm{MIC}$ ) as predictors of outcome for cefepime and ceftazidime in serious bacterial infections. Int J Antimicrob Agents 31: 345-351.

27. Li C, Du X, Kuti JL, Nicolau DP (2007) Clinical pharmacodynamics of meropenem in patients with lower respiratory tract infections. Antimicrob Agents Chemother 51: 1725-1730. 